\title{
To Evaluate the Influence of Implant Length on Stress Distribution of Osseointegrated Implant: A Three- Dimensional Finite Element Analysis: An In Vitro Study
}

\author{
Neha Jindal ${ }^{1}$ Manjit Kumar ${ }^{1} \quad$ Shailesh Jain ${ }^{1} \quad$ Raman $^{1} \quad$ Navjot Kaur $^{1}$ \\ ${ }^{1}$ Department of Prosthodontics, Bhojia Dental College, Solan, \\ Address for correspondence Manjit Kumar, MDS, Department of \\ Himachal Pradesh, India \\ Prosthodontics, Bhojia Dental College, Baddi 173205, Distt. Solan, \\ Himachal Pradesh, India (e-mail: manjitkiran@yahoo.co.in.com).
}

Dent J Adv Stud 2018;6:97-105

\begin{abstract}
Finite element analysis is a technique for obtaining a solution to a complex mechanical problem by dividing the problem domain into a collection of much smaller and simpler domains (elements) in which the field variables can be interpolated with the use of shape functions. An overall approximated solution to the original problem is determined based on variational principles. Finite element analysis can provide a nondestructive system for quantifying stresses generated at the various interfaces of similar or dissimilar material. The finite element method also allows the study of the internal state of stress of components as well as stress patterns in two or more dissimilar materials adjacent to each other without affecting their independent behavior. This method is therefore ideally suitable for the biomechanical analysis of orthopedic, cardiovascular, and dental structures. In this study, implants of different length were numerically analyzed using bone-implant models developed from computed tomography-generated images of the mandible with osseointegrated implants. The impact of various lengths

Keywords

- dental implant

- finite element analysis study

- implant length

- masticatory load

- stress analysis

- von Mises stress on stress distribution was examined using implants with a length of 8,10 , and $13 \mathrm{~mm}$ in mandibular first molar region under axial load of $100 \mathrm{~N}$ and buccolingual load of $50 \mathrm{~N}$. All materials were assumed to be linearly elastic and isotropic. The Statistical Package for the Social Sciences software package was used for statistical analysis. Maximum von Mises stresses were located around the implant neck. It was demonstrated that there was statistically nonsignificant decrease in von Mises stress as the implant length increased. Within the limitations of this study, there was statistically nonsignificant decrease in von Mises stress as the implant length increased.
\end{abstract}

\section{Introduction}

It was possible to fix dental prostheses with intraosseous implants after the initiation of the concept of Osseo integration. ${ }^{1}$ Success rate for implants in the mandible and maxilla have been reported as $95 \%$ and $90 \%$, respectively, along with continued marginal bone loss over two decades. Marginal bone loss is seen to be due to poor oral hygiene and biomechanical factors. ${ }^{2}$

received

October 30, 2018 accepted after revision

November 30, 2018

published online

January 29, 2019
Dental implant transfers masticatory forces within jaws to the supporting osseous tissues. Many authors have emphasized the significance of various factors such as loading type, length and diameter of implants, topographic characteristics of implant, prosthesis type, and other biomechanical factors. ${ }^{3}$

Finite element analysis (FEA) divides the problem domain into a collection of smaller and simpler domains in which the field variables can be interpolated by using shape functions.

(C2018 Bhojia Dental College and

License terms

Hospital affiliated to Himachal

Pradesh University

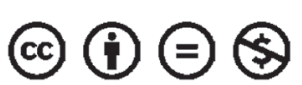


A comprehensive approximated solution to the problem is determined based on ever-changing principles. ${ }^{4}$

FEA helps measure stresses generated at the various interfaces of similar or dissimilar material. It also allows the study of the internal state of stress of components as well as stress patterns in two or more dissimilar materials adjacent to each other without affecting their independent behavior. This method is therefore ideally suitable for the biomechanical analysis of cardiovascular and dental structures. ${ }^{5}$

In this process, the structure of interest is divided into nodes and elements to create a mesh that is referred to as discretization, and computationally a system of simultaneous equations is generated to relate all the forces and displacement at nodes. Hence, the problem is converted into matrix algebra, and its solution is represented via displacements, strains, and stresses within a structure. The axi-symmetric techniques along with two- and three-dimensional (3D) models can be used although the 3D model can provide a more accurate prediction of stress distribution. ${ }^{6}$

Previously substantial extensive researches have been attempted on the characteristics of stress distribution in bone surrounding implants. However, FEA has not been extensively used to investigate the role of various implant dimensions on stress distribution in mandibular molar areas and biomechanical behavior of the mandibular cancellous bone when in contiguity to the cortical bone.

The aim of this article is to evaluate the stress in periimplant area of the cancellous bone by different lengths of implant in the molar region of the mandible, to understand the impact of implant length on stress distribution, and to understand the overload of clinically evidenced crestal bone loss at the implant neck in mandibular molar peri-implant region using 3D FEA.

\section{Materials and Methods}

\section{Materials}

- MIS implants (length 8, 10,13 mm)

- Dried specimen of human partially edentulous mandible (with missing first molars) used for coordinate measuring machine scanning

- Finite element software ANSYS v. 18.1 (CADD Centre, Chandigarh, India)

\section{Methodology}

To accomplish this, solid models of tapered threaded dental implants with lengths of 8,10 , and $13 \mathrm{~mm}$ were developed and studied. These dimensions were selected on the basis of most routinely used implants. The finite element software ANSYS v. 18.1 was used to merge the bone-implant model and to generate and solve the discrete finite element meshes. Computed tomographic (CT) images of the human mandible were used to develop 3D models of the mandibular molar segment. In the model, implant was positioned in vertical direction in the molar region. The whole bone was considered to be a homogeneous, isotropic material with the character of cortical bone. Structure of cancellous bone is altered after osseointegration, and bone-implant interface resembles cortical bone.
The geometric models of implant and mandibular bone were generated ( $\bullet$ Figs. 1, 2) using CMM $(-$ Fig. $\mathbf{3})$ and then imported. The process of converting geometric model into finite element model called meshing was performed. The finite element model consisted of nodes and elements as shown ( - Tables 1, 2).

The assembled finite element model of inserted implant in bone (-Fig. 4), then imported into ANSYS 18.1 (ANSYS 18.1, Inc., Canonburg, PA) software for analysis. The material properties of implant and bone were entered in the preprocessing stage ( $\mathbf{-}$ Table $\mathbf{3}$ ).

The applied force and boundary conditions were applied (-Fig. 5). Postprocessing the results and capturing the von Mises stress and strain contours of each individual section in the system was done (-Figs. 6-9). Preprocessing, solving, and postprocessing are three separate stages in the ANSYS software. The force simulated was in the same direction as in occlusion that is in axial and buccolingual directions of $100 \mathrm{~N}$ and $50 \mathrm{~N}$, respectively, which was loaded to the head of implant.

After application of force, the magnitude was analyzed by ANSYS Workbench (ANSYS 18.1, Inc., United States) a 3D FEA program. A color scale was used to assess quantitatively the stress distribution in the bone (cortical and cancellous) and implant interface. Red and blue indicate areas with the highest and lowest stress and strain, respectively (-Figs. 5-9).

\section{Constraints and Loads}

The models were constrained in all directions at the nodes on the distal-end surface of the bone segment. Forces of $100 \mathrm{~N}$ and $50 \mathrm{~N}$ were applied axially and buccolingually, respectively, to the occlusal node at the center of the implant. The maximum and minimum equivalent von Mises (EQV) stresses contour at cortical bone, cancellous bone, and implants were calculated.

\section{Results}

This study was aimed to assess the amount of stress/strain field developing at implant and bone interface with implant length of 8,10 , and $13 \mathrm{~mm}$ in mandibular first molar

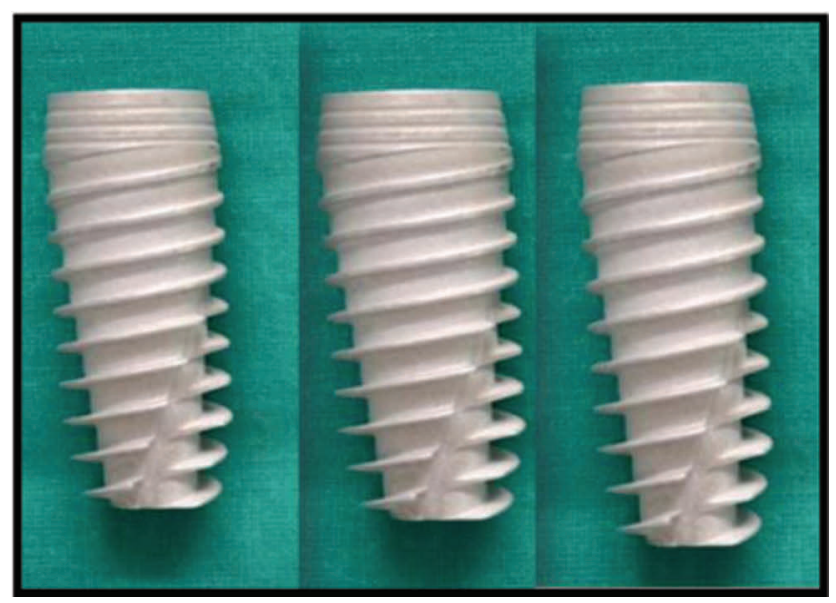

Fig. 1 MIS implants $(8,10,13 \mathrm{~mm})$. 


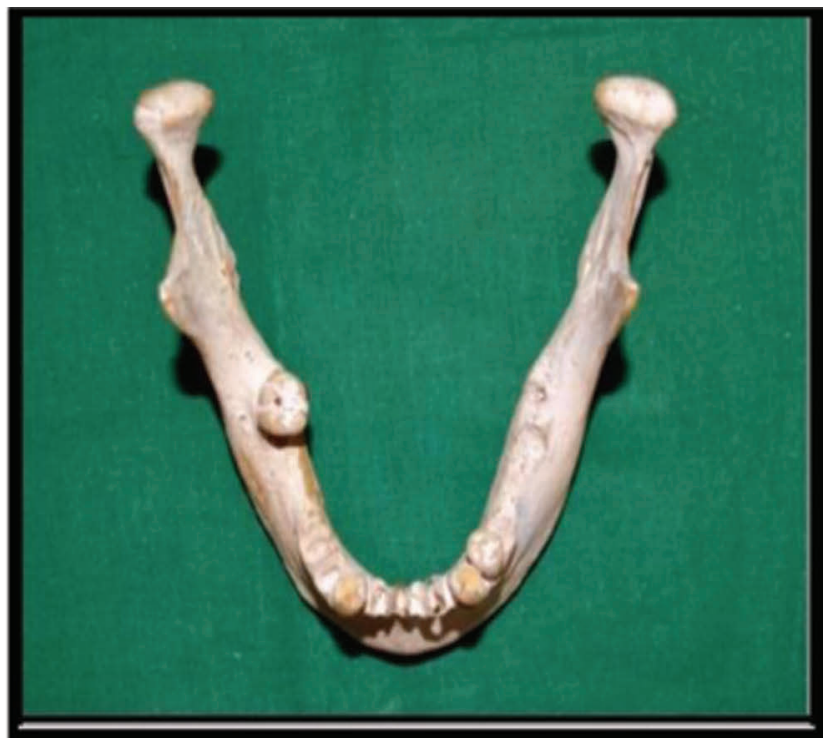

Fig. 2 Dried specimen of human partially edentulous mandible (with missing first molars).

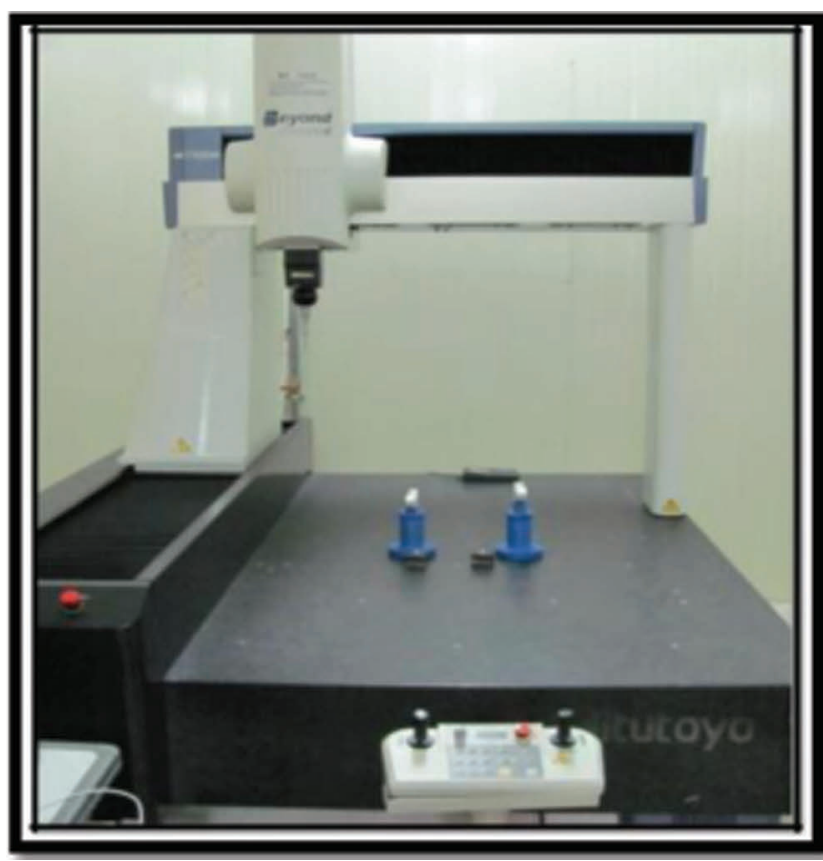

Fig. 3 Coordinate measuring machine.

Table 1 Number of element and nodes generated for each model

\begin{tabular}{|l|l|l|}
\hline Model & No. of elements & No. of nodes \\
\hline Implant & 9,756 & 17,706 \\
\hline Bone & 80,651 & 145,973 \\
\hline
\end{tabular}

region under axial and buccolingual loads of $100 \mathrm{~N}$ and $50 \mathrm{~N}$, respectively. A color scale showing von Mises stress distribution values were used to assess quantitatively the stress distribution in the bone and implant interface (-Figs. 6-9). Red and blue indicate areas with highest and lowest stress, respectively.
Table 2 Number of elements and nodes used for model generation of different length of implant and bone

\begin{tabular}{|l|l|l|}
\hline Model & Elements & Nodes \\
\hline 8 -mm implant & 4,283 & 7,760 \\
\hline 10 -mm implant & 5,473 & 9,946 \\
\hline $13-m m$ implant & 6,663 & 12,132 \\
\hline Cortical bone & 18,427 & 36,566 \\
\hline Cancellous bone & 23,330 & 38,604 \\
\hline
\end{tabular}

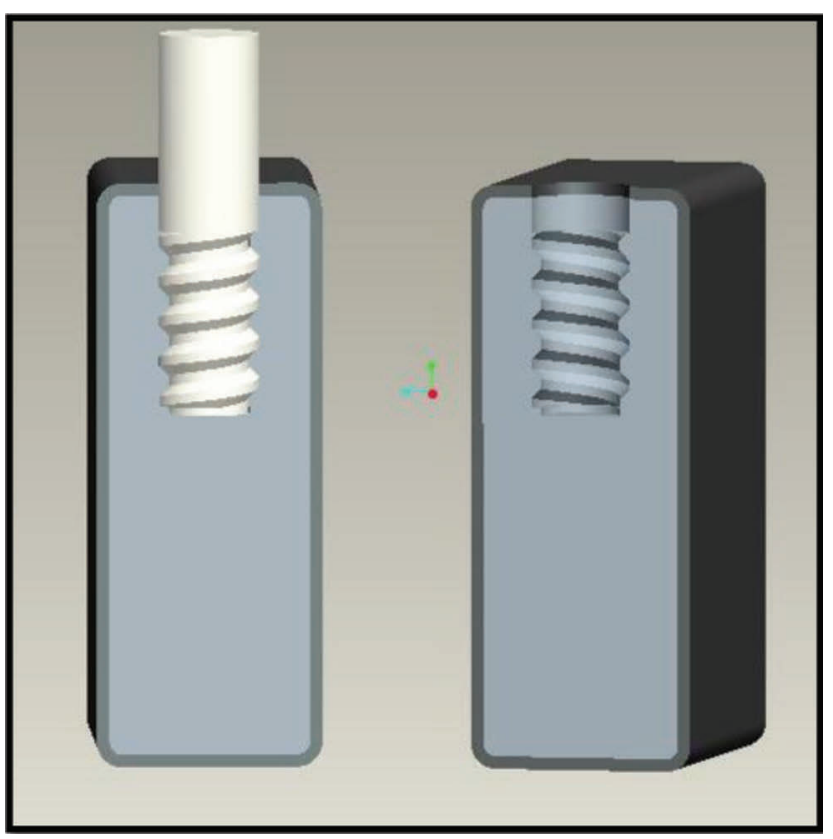

Fig. 4 Implant model inserted in the bone model.

Table 3 Physical properties of component of bone model

\begin{tabular}{|l|l|l|}
\hline Material & Cortical bone & Cancellous bone \\
\hline $\begin{array}{l}\text { Elastic modulus } \\
(\mathrm{MPa})\end{array}$ & 14700 & 1470 \\
\hline Poisson's ratio & 0.30 & 0.30 \\
\hline
\end{tabular}

The maximum von Mises stress values obtained from scale in cortical and cancellous bones and implant interface were recorded and compared with the help of - Tables 4-8 and Graphs 1-4.

Further, it was concluded that when implant length was changed from 8,10 , and $13 \mathrm{~mm}$ in the mandibular first molar region with the help of ANSYS 18.1 software and data were analyzed statistically, the stresses with axial and buccolingual loads generated in all body model were within the tolerable limit whereas the effect of implant length was nonsignificant.

\section{Statistical Methods}

Repeated measures ANOVA (analysis of variance) test was used to statistically assess the impact of implant length on stress distribution of osseointegrated implant. The result was 

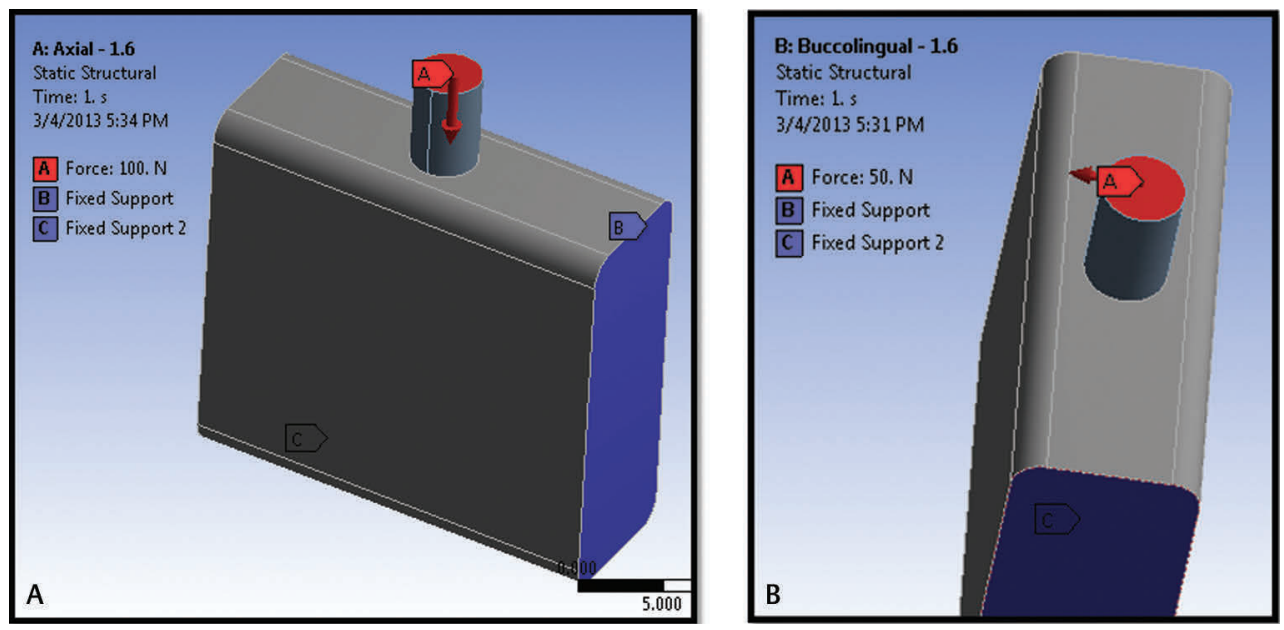

Fig. 5 Finite element model and definition of load direction. (A) Axial load of 100 N. (B) Buccolingual load of 50 N.
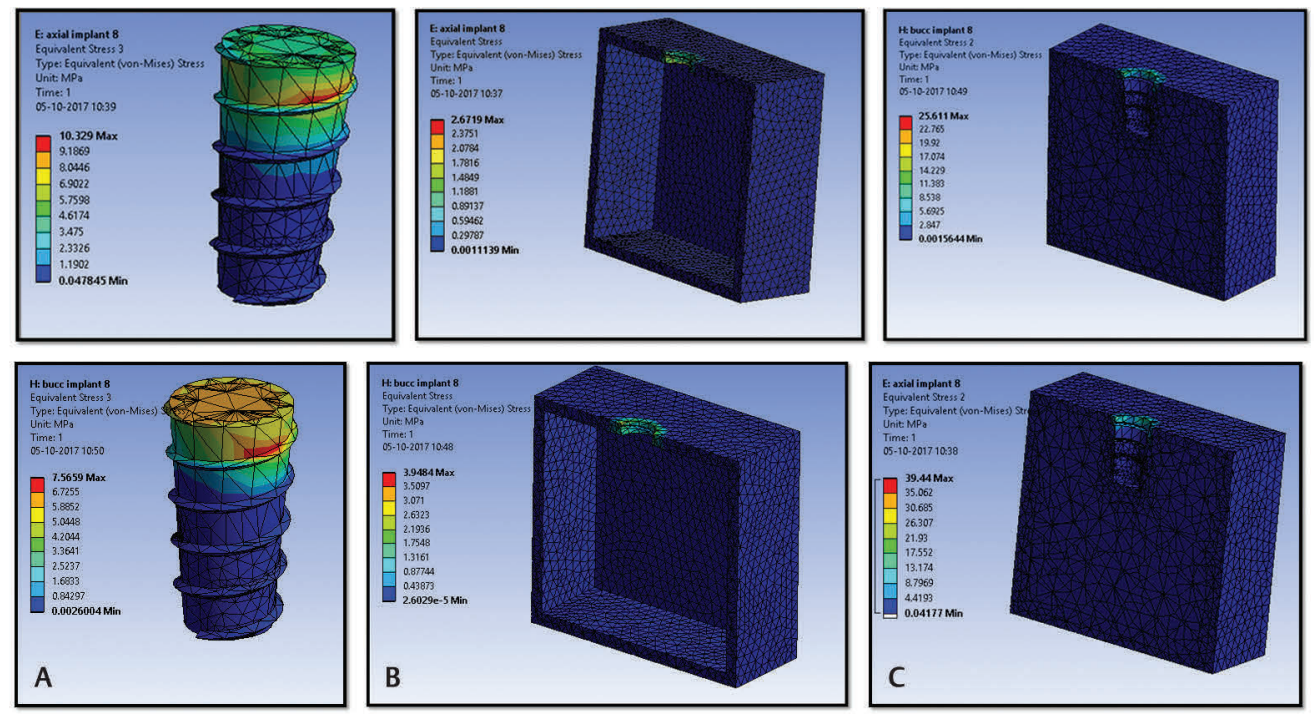

Fig. 6 (A) An 8-mm implant model showing stress distribution on implant in cancellous bone when axial and buccolingual forces are loaded. (B) Cortical bone model showing stress distribution on cortical bone when axial and buccolingual forces are loaded on 8-mm implant. (C) Cancellous bone model showing stress distribution on cancellous bone when axial and buccolingual forces are applied on 8-mm implant.

confirmed by ANOVA for all the parameters. To determine whether variance in implant length had an effect on clinical parameters, stepwise ANOVA was used. In this study, it was accomplished that these stresses were within tolerable limit, and thus the effect of different implant length in the mandibular first molar region was nonsignificant $(p<0.05)$. The maximum von Mises stress values with axial force on implants were observed to be $5.18 \mathrm{MPa}$ for $8-\mathrm{mm}, 5.07 \mathrm{MPa}$ for $10-\mathrm{mm}$, and $5.07 \mathrm{MPa}$ for $13-\mathrm{mm}$ implant. The maximum von Mises stress values with buccolingual force on implants were observed to be $3.78 \mathrm{MPa}$ for 8-mm, 4.92 MPa for 10-mm and 4.34 MPa for 13-mm implant (-Table 4, Graph 1).

The maximum von Mises strain values with axial force on implants were observed to be $1.26 \mathrm{~mm} / \mathrm{mm}$ for $8-\mathrm{mm}, 1.33 \mathrm{~mm} / \mathrm{mm}$ for $10-\mathrm{mm}$, and $1.10 \mathrm{~mm} / \mathrm{mm}$ for 13-mm implant. The maximum von Mises strain values with buccolingual force on implants were observed to be $0.893 \mathrm{~mm} / \mathrm{mm}$ for $8-\mathrm{mm}, 0.985 \mathrm{~mm} / \mathrm{mm}$ for $10-\mathrm{mm}$, and $0.921 \mathrm{~mm} / \mathrm{mm}$ for $13-\mathrm{mm}$ implant (-Table 5, Graph 2).

The maximum von Mises stress values with axial force on cortical bone were observed to be $1.33 \mathrm{MPa}$ for $8-\mathrm{mm}, 1.08 \mathrm{MPa}$ for $10-\mathrm{mm}$, and $1.29 \mathrm{MPa}$ for $13-\mathrm{mm}$ implant. The maximum von Mises stress values with buccolingual force on cortical bone were observed to be $2.23 \mathrm{MPa}$ for $8-\mathrm{mm}, 1.84 \mathrm{MPa}$ for $10-\mathrm{mm}$, and 2.18 MPa for 13-mm implant (-Table 6, Graph 3).

The maximum von Mises strain values with axial force on cortical bone were observed to be $0.576 \mathrm{~mm} / \mathrm{mm}$ for $8-\mathrm{mm}, 0.845 \mathrm{~mm} / \mathrm{mm}$ for $10-\mathrm{mm}$, and $0.924 \mathrm{~mm} / \mathrm{mm}$ for $13-\mathrm{mm}$ implant. The maximum von Mises strain values with buccolingual force on cortical bone were observed to be $0.177 \mathrm{~mm} / \mathrm{mm}$ for $8-\mathrm{mm}, 0.218 \mathrm{~mm} / \mathrm{mm}$ for $10-\mathrm{mm}$, and $0.192 \mathrm{~mm} / \mathrm{mm}$ for 13-mm implant (-Table 7, Graph 4). 

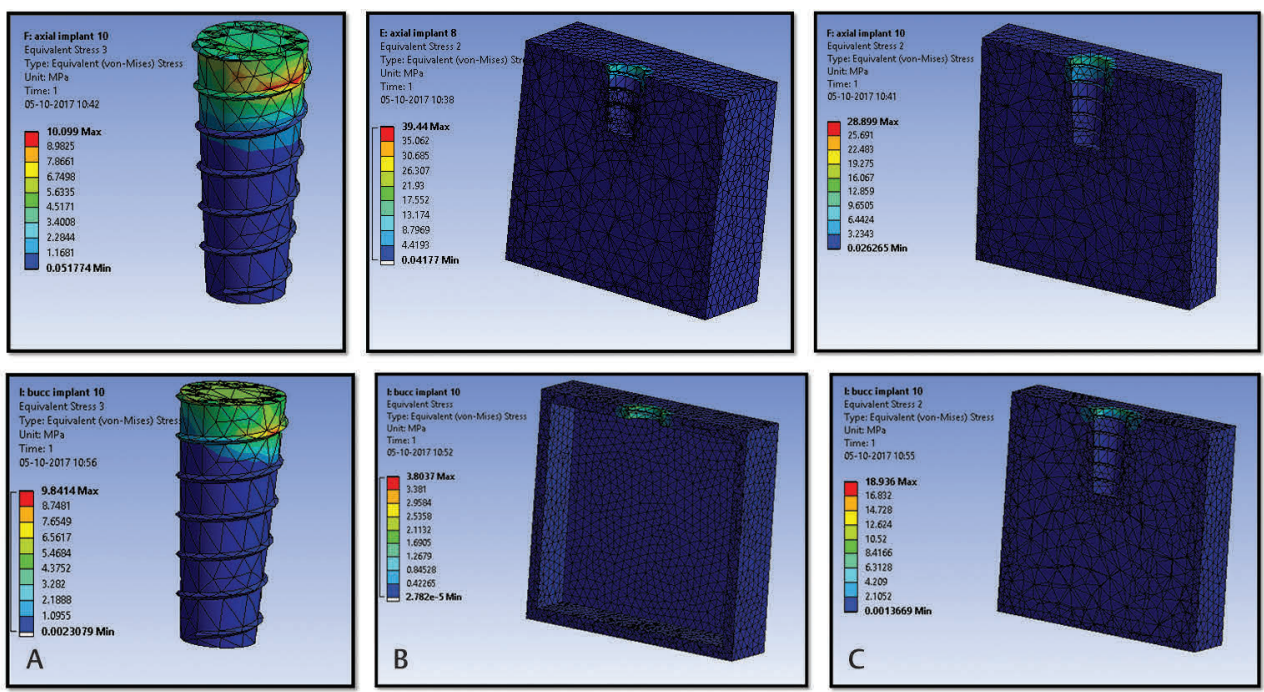

Fig. 7 (A) A 10-mm implant model showing stress distribution on implant in cancellous bone when axial and buccolingual forces are applied. (B) Cortical bone model showing stress distribution on cortical bone when axial and buccolingual forces are applied on 10-mm implant. (C) Cancellous bone model showing stress distribution on cancellous bone when axial and buccolingual forces are applied on 10-mm implant.
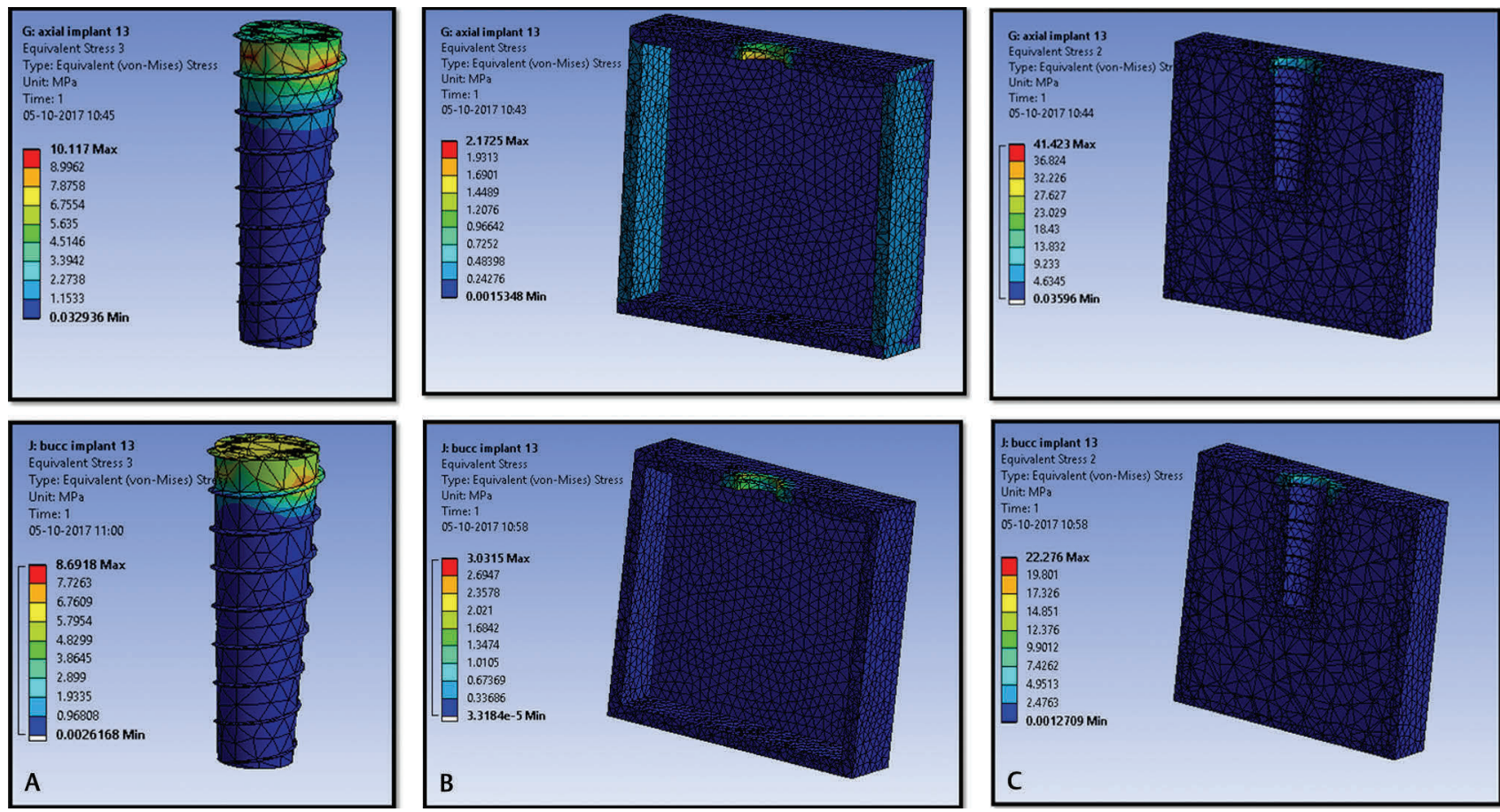

Fig. 8 (A) A 13-mm implant model showing stress distribution on implant in cancellous bone when axial and buccolingual forces are applied.

(B) Cortical bone model showing stress distribution on cortical bone when axial and buccolingual forces are loaded on 13-mm implant.

(C) Cancellous bone model showing stress distribution on cancellous bone when axial and buccolingual forces are loaded on 13-mm implant.

\section{Discussion}

Implant biomechanics is a significant factor in longevity of dental implants. The ability of implant to transfer physiologic loads within a range of 100 to $3,000 \mu \mathrm{m}$ strains to the surrounding bone depends on implant biomechanics. ${ }^{8}$ Clinical studies have stated remarkable bone loss around the neck of failed implants, and different theories have been stated to interpret this bone reaction. Unfavorable loading has been seen to cause increased stress in bone around implant leading to bone loss, and thus implant failure as demonstrated by various animal experiments ${ }^{9}$ and clinical studies. Thus, it is important to evaluate the stress/strain in bone and their relation to various parameters of implant and bone. This study used the finite element method to evaluate the effect of implant length on stress in bone and implant.

In a comparative analysis, complex reality can be simplified assuming that proportions and relative effect accurately reflect reality. Because simulation of the whole mandibular body is very magnificent, smaller models have been offered for parameter studies. ${ }^{10}$ Here in this analysis, a portion of 

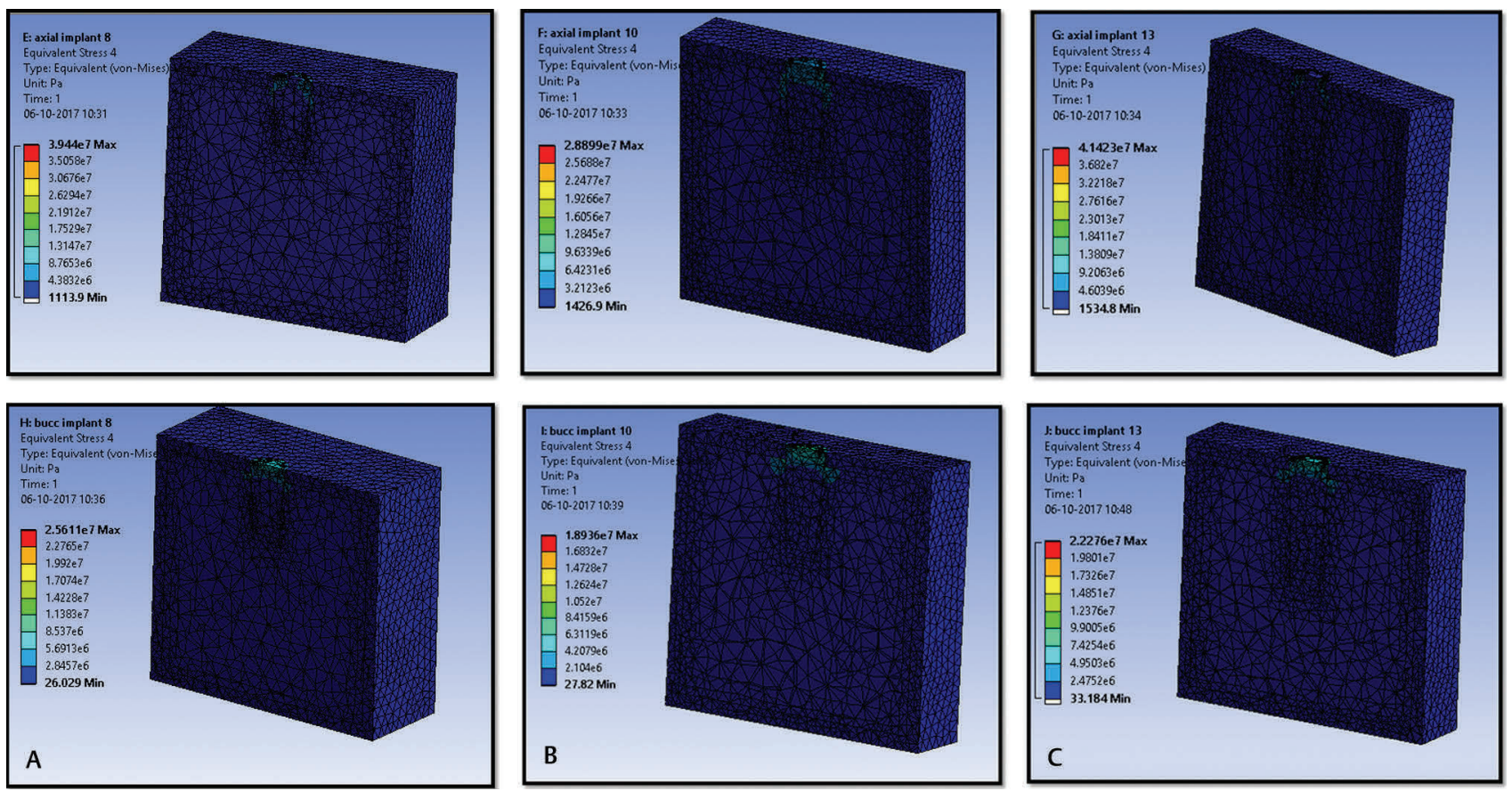

Fig. 9 (A) All body model showing stress distribution on implant bone interface when axial and buccolingual forces are loaded on an 8-mm implant. (B) All body model showing stress distribution on implant bone interface when axial and buccolingual forces are loaded on a 10-mm implant. (C) All body model showing stress distribution on implant bone interface when axial and buccolingual forces are loaded on a 13-mm implant.

Table 4 Descriptive von Mises stress values with axial $(100 \mathrm{~N})$ and buccolingual loads $(50 \mathrm{~N})$ on different length of implants inserted in bone (without the effect of cortical and cancellous bone)

\begin{tabular}{|l|l|l|l|l|}
\hline Variable & Implant $(\mathbf{m m})$ & Mean & Standard deviation & Standard error mean \\
\hline \multirow{5}{*}{ Stress-axial } & 8 & 5.18 & 3.45 & 1.09 \\
\cline { 2 - 5 } & 10 & 5.07 & 3.37 & 1.06 \\
\cline { 2 - 5 } & 13 & 5.07 & 3.39 & 1.07 \\
\hline \multirow{3}{*}{ Stress-buccolingual } & 8 & 3.78 & 2.54 & 0.804 \\
\cline { 2 - 5 } & 10 & 4.92 & 3.30 & 1.04 \\
\cline { 2 - 5 } & 13 & 4.34 & 2.92 & .924 \\
\hline
\end{tabular}

Table 5 Descriptive von Mises stress values with axial $(100 \mathrm{~N})$ and buccolingual loads $(50 \mathrm{~N})$ on cortical bone with different lengths of implants (without cancellous bone)

\begin{tabular}{|l|l|l|l|l|}
\hline Variable & Implant $(\mathbf{m m})$ & Mean & Standard deviation & Standard error mean \\
\hline \multirow{4}{*}{ Stress-axial } & 8 & 1.33 & 0.898 & 0.284 \\
\cline { 2 - 5 } & 10 & 1.08 & 0.730 & 0.230 \\
\cline { 2 - 5 } & 13 & 1.29 & 0.867 & 0.274 \\
\hline \multirow{3}{*}{ Stress-buccolingual } & 8 & 2.23 & 1.14 & 0.360 \\
\cline { 2 - 5 } & 10 & 1.84 & 1.01 & 0.319 \\
\cline { 2 - 5 } & 13 & 2.18 & 1.11 & 0.351 \\
\hline
\end{tabular}

bone was modeled to simulate the posterior region of the mandible.

The shape of the cancellous bone was simplified to a rectangular block to facilitate the modeling of the same amount of bone for the three types of implants, while only the outer edges of the cortical bone were rounded.

In the studies of the whole mandible, in which convergence tests with mesh refinements have been done, models with more than 13,720 or 10,420 nodes showed convergent results. ${ }^{11} \mathrm{~A}$ fine mesh is important to accomplish a precise model. The main advantage of small, simplified models is that they allow for finer meshing with hexahedron elements. Thus in this study, only a mandibular segment with an extremely fine mesh around the implant (areas of high stress) was modeled. This resulted in a model consisting of 105,008 nodes, a number that was considered to ensure a sufficiently fine mesh for the given geometry, and no further mesh refinement was performed. 
Table 6 Descriptive von Mises stress values with axial $(100 \mathrm{~N})$ and buccolingual loads $(50 \mathrm{~N})$ on cancellous bone with different lengths of implants (without cortical bone)

\begin{tabular}{|l|l|l|l|l|}
\hline Variable & Implant $(\mathbf{m m})$ & Mean & Standard deviation & Standard error mean \\
\hline \multirow{3}{*}{ Stress-axial } & 8 & 19.74 & 13.25 & 4.19 \\
\cline { 2 - 5 } & 10 & 14.46 & 9.713 & 3.07 \\
\cline { 2 - 5 } & 13 & 20.7 & 13.9 & 4.40 \\
\hline \multirow{3}{*}{ Stress-buccolingual } & 8 & 1.28 & 8.61 & 2.72 \\
\cline { 2 - 5 } & 10 & 9.46 & 6.36 & 2.01 \\
\cline { 2 - 5 } & 13 & 1.11 & 7.49 & 2.36 \\
\hline
\end{tabular}

Table 7 Descriptive von Mises stress values with axial $(100 \mathrm{~N})$ and buccolingual loads $(50 \mathrm{~N})$ on all body models with varying lengths of implants (with cortical and cancellous bone)

\begin{tabular}{|l|l|l|l|l|}
\hline Variable & Implant $(\mathbf{m m})$ & Mean & Standard deviation & Standard error mean \\
\hline \multirow{3}{*}{ Stress-axial } & 8 & 1.14 & 351.1 & 111.0 \\
\cline { 2 - 5 } & 10 & 1.45 & 450.1 & 142.3 \\
\cline { 2 - 5 } & 13 & 1.36 & 430.18 & 133.1 \\
\hline Stress-buccolingual & 8 & 5.42 & 7.59 & 2.40 \\
\cline { 2 - 5 } & 10 & 5.62 & 8.17 & 2.58 \\
\cline { 2 - 5 } & 13 & .921 & 2.26 & 0.716 \\
\hline
\end{tabular}

Table 8 Intergroup comparison of stress of all body models of all the groups

\begin{tabular}{|c|c|c|c|c|c|}
\hline Variable & Group & Sum of Squares & Mean square & $\mathrm{F}$ & Sig. \\
\hline \multirow[t]{3}{*}{ Stress-axial } & Between groups & $9,615.716$ & $4,807.858$ & \multirow[t]{3}{*}{0.026} & \multirow[t]{3}{*}{0.9} \\
\hline & Within groups & $5,043,109.079$ & $186,781.818$ & & \\
\hline & Total & $5,052,724.794$ & & & \\
\hline \multirow[t]{3}{*}{ Stress-buccolingual } & Between groups & 8.847 & 4.424 & \multirow[t]{3}{*}{0.060} & \multirow[t]{3}{*}{0.1} \\
\hline & Within groups & $1,978.032$ & 73.260 & & \\
\hline & Total & $1,986.879$ & & & \\
\hline
\end{tabular}

${ }^{a}$ Non significant

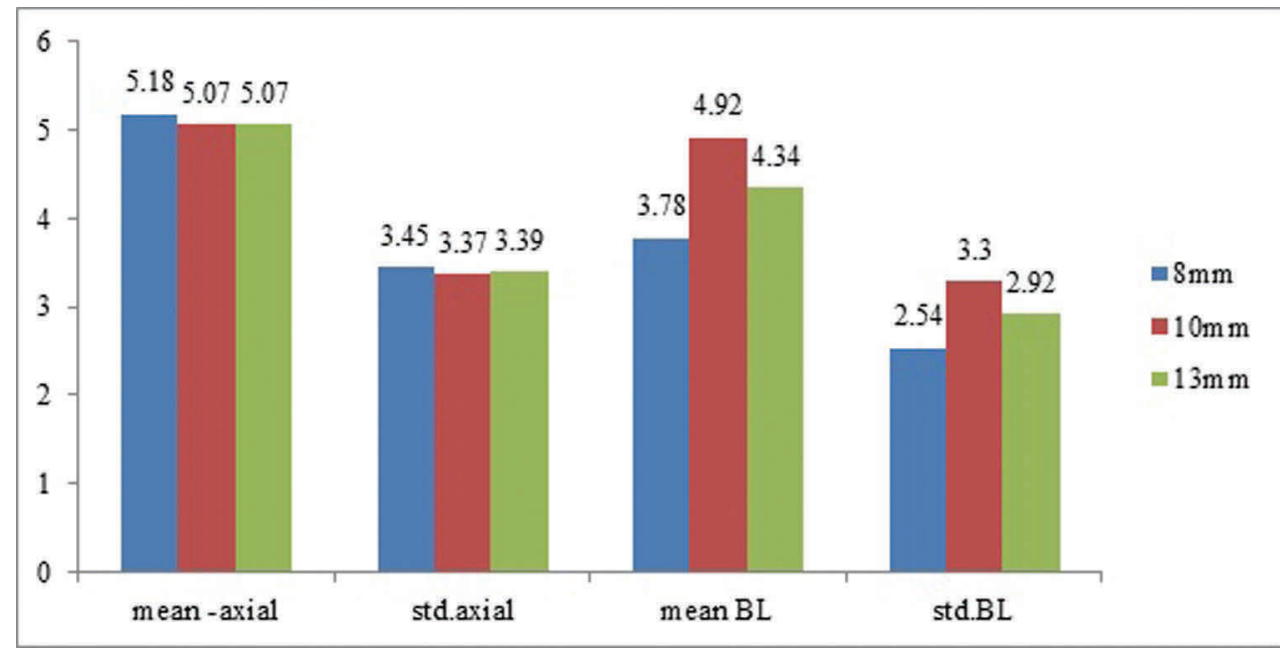

Graph 1 Descriptive von Mises stress values with axial (100 N) and buccolingual loads ( $50 \mathrm{~N})$ on different lengths of implants inserted in bone.

When only cancellous bone was considered in contact with implant-bone interface, without the contribution of cortical bone, the maximum EQV stress came out to be
19.74 MPa with 8-mm implant that further decreased to 14.46 MPa with 10-mm implant, but on further increasing the length to $13 \mathrm{~mm}$, the stress increased further 


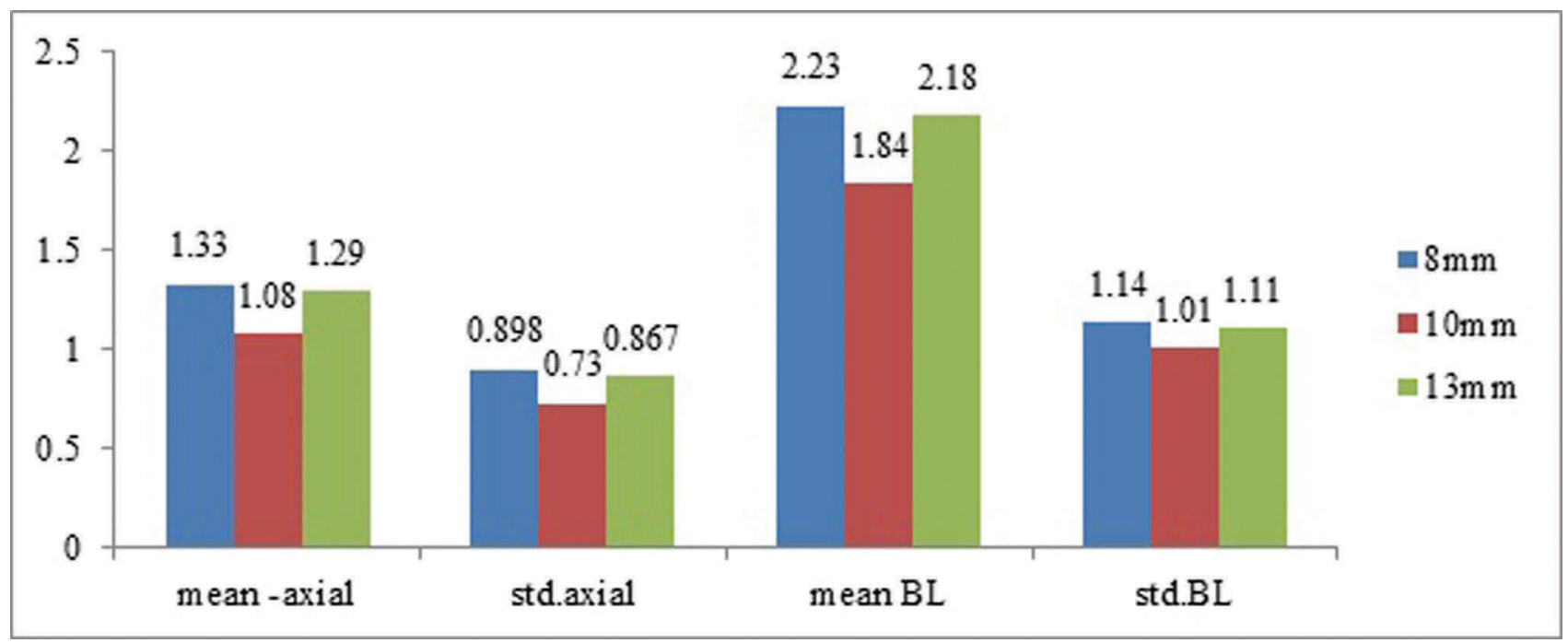

Graph 2 Descriptive von Mises stress values with axial (100 N) and buccolingual loads ( $50 \mathrm{~N})$ on cortical bone with different lengths of implants.

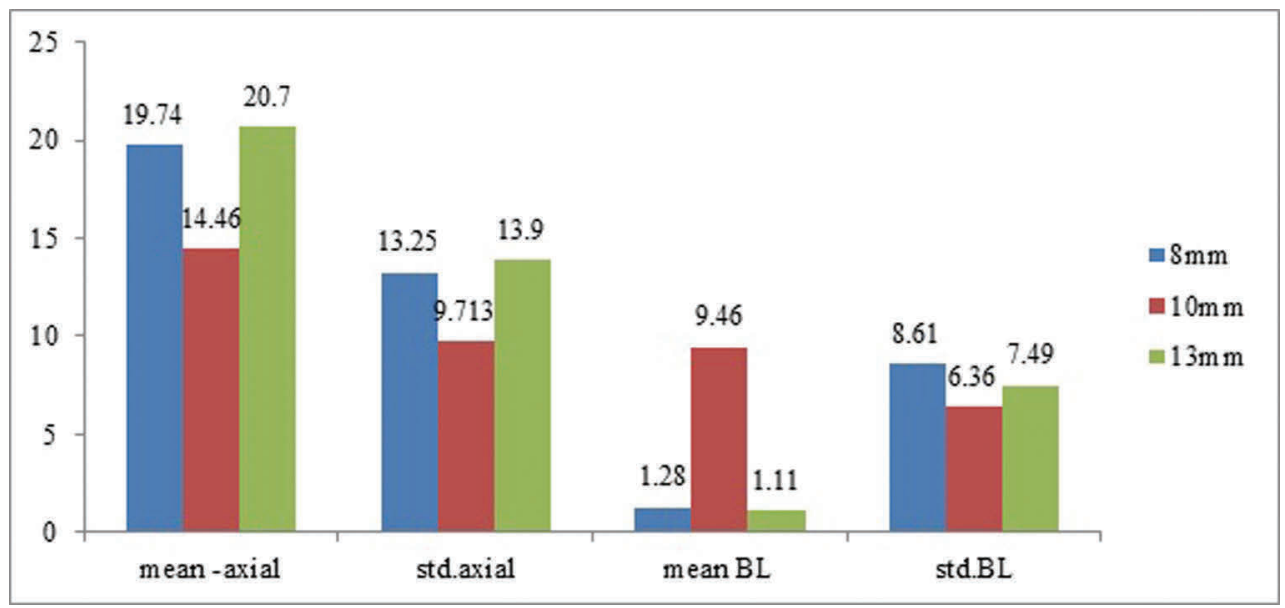

Graph 3 Descriptive von Mises stress values with axial (100 N) and buccolingual loads (50 N) on cancellous bone with different lengths of implants.

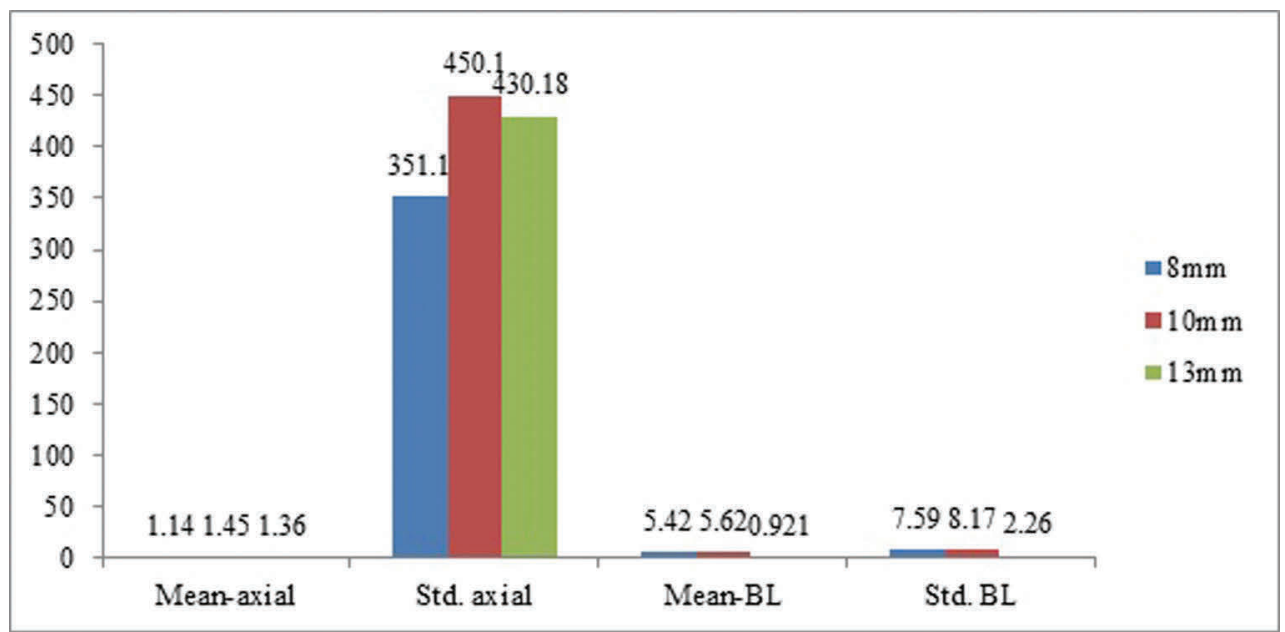

Graph 4 Descriptive von Mises stress values with axial (100 N) and buccolingual loads ( $50 \mathrm{~N})$ on all body models with varying lengths of implants.

up to 20.7 MPa that is almost to the level of 8-mm-long implant. These findings are in contrast of what Baggi et al ${ }^{12}$ observed. In his research, with increased implant length, stress gradients seemed to decrease at the cancellous peri-implant region.
The cancellous region withstood the axial load less efficiently, and the load was remarkably displaced downward. Thus, high or moderate stresses were found over large areas at the bone-implant interface, and highest stresses were located around the implant base. In these instances, 
the presence of threads had a favorable influence on force transmission to bone resulting in dissipating force.

Under buccolingual load, in most instances, stress was concentrated in the cancellous bone around the implant neck, near the fulcrum of implant bending. For this reason, differences in implant length in the lower part of the implant did not influence the strain distribution.

When only the cortical bone was considered in contact with implant-bone interface, without the contribution of the cancellous bone, the maximum EQV stress came out to be $1.33 \mathrm{MPa}$ with $8-\mathrm{mm}$ implant that further decreased to $1.08 \mathrm{MPa}$ with $10-\mathrm{mm}$ implant, but on further increasing the length to $13 \mathrm{~mm}$, the stress increased further up to $1.29 \mathrm{MPa}$ that is almost to the level of 8-mm-long implant.

Because the cortical bone had a much higher elastic modulus than the cancellous bone, it was the main load-carrying member for all cancellous bone qualities, regardless of load direction. Higher stress concentration around the implant neck was also found in many 3D FEAs. Implant length did not greatly influence stress distribution in the cortical bone, probably because the implant neck had the same shape and diameter in all models and transferred the stress similarly to the surrounding bone.

\section{Summary}

Under axial load, maximum EQV stress on implant decreased from 8 to $10 \mathrm{~mm}$ and remained constant from 10 to $13 \mathrm{~mm}$, and under buccolingual load, maximum EQV stress on implant increased from 8 to $10 \mathrm{~mm}$ and again decreased from 10 to $13 \mathrm{~mm}$. Under axial load, maximum EQV strain on implant increased from 8 to $10 \mathrm{~mm}$ and again decreased from 10 to $13 \mathrm{~mm}$, and under buccolingual load, maximum EQV strain on implant increased from 8 to $10 \mathrm{~mm}$, but it again decreased from 10 to $13 \mathrm{~mm}$.

Under axial load, maximum EQV stress on the cortical bone decreased from 8 to $10 \mathrm{~mm}$ and again increased from 10 to $13 \mathrm{~mm}$, and under buccolingual load, maximum EQV stress on the cortical bone increased from 8 to $10 \mathrm{~mm}$ and again decreased from 10 to $13 \mathrm{~mm}$. Under axial load, maximum EQV strain on the cortical bone increased as the length of the implant increased, and under buccolingual load, maximum EQV strain on implant increased from 8 to $10 \mathrm{~mm}$, but it again decreased from 10 to $13 \mathrm{~mm}$.

Under axial load, maximum EQV stress on the cancellous bone decreased from 8 to $10 \mathrm{~mm}$ and further increased from 10 to $13 \mathrm{~mm}$, and under buccolingual load, maximum EQV stress on implant increased from 8 to $10 \mathrm{~mm}$, but it again decreased from 10 to $13 \mathrm{~mm}$. Under axial load, maximum $\mathrm{EQV}$ strain on implant increased from 8 to $10 \mathrm{~mm}$ and again decreased from 10 to $13 \mathrm{~mm}$, and under buccolingual load, maximum EQV strain on implant increased from 8 to $10 \mathrm{~mm}$, but it again decreased from 10 to $13 \mathrm{~mm}$.

Under axial load, maximum EQV stress on all body model (cortical and cancellous) increased from 8 to $10 \mathrm{~mm}$, but it again decreased from 10 to $13 \mathrm{~mm}$, and under buccolingual load also, maximum EQV stress on all body model (cortical and cancellous) increased from 8 to $10 \mathrm{~mm}$ and again decreased from 10 to $13 \mathrm{~mm}$.

\section{Conclusion}

Maximum EQV stress and strain in the cancellous and in cortical bones occurred around neck of implants, confirming the importance of crestal bone loss due to increased occlusal load and importance of presurgical diagnosis for implant long-term prognosis.

Under axial and buccolingual loads, the influence of implant length on bone stress and strain appeared to be nonsignificant.

Within limitations of this study, the length of implant has minimal clinical significance as far as stress and strain are concerned as compared with other factors.

There is still further scope for improving the clinical significance of this study by additional parameters that are diameter of implant, number of threads of implant, splinted implants, and comparison between different densities of bone.

\section{Funding \\ None. \\ Conflict of Interest \\ None declared.}

\section{References}

1 Tripathi R. Application of Finite Element Analysis in Dentistry. 3rd ed. PA, NJ: Prentice Hall; 2002:1-22

2 Petrie CS, Williams JL. Comparative evaluation of implant designs: influence of diameter, length, and taper on strains in the alveolar crest. A three-dimensional finite-element analysis. Clin Oral Implants Res 2005;16(4):486-494

3 Geng JP, Tan KBC, Liu GR. Application of finite element analysis in implant dentistry: a review of the literature. J Prosthet Dent 2001;85(6):585-598

4 Sevimay M, Turhan F, Kiliçarslan MA, Eskitascioglu G. Threedimensional finite element analysis of the effect of different bone quality on stress distribution in an implant-supported crown. J Prosthet Dent 2005;93(3):227-234

5 Kong L, Hu K, Li D, et al. Evaluation of the cylinder implant thread height and width: a 3-dimensional finite element analysis. Int J Oral Maxillofac Implants 2008;23(1):65-74

6 Guan H, van Staden R, Loo YC, Johnson N, Ivanovski S, Meredith $\mathrm{N}$. Influence of bone and dental implant parameters on stress distribution in the mandible: a finite element study. Int J Oral Maxillofac Implants 2009;24(5):866-876

7 Thirupathi RC. Introduction to Finite Elements in Engineering. 3rd ed. PA, NJ: Prentice Hall; 2005:1-15

8 Carter DR, Van Der Meulen MC, Beaupré GS. Mechanical factors in bone growth and development. Bone 1996;18 (1, Suppl):5S-10S

9 Isidor F. Loss of osseointegration caused by occlusal load of oral implants. A clinical and radiographic study in monkeys. Clin Oral Implants Res 1996;7(2):143-152

10 Mericske-Stern R. Overdentures with roots or implants for elderly patients: a comparison. J Prosthet Dent 1994;72(5):543-550

11 Hart RT, Hennebel VV, Thongpreda N, Van Buskirk WC, Anderson RC. Modeling the biomechanics of the mandible: a three-dimensional finite element study. J Biomech 1992;25(3):261-286

12 Baggi L, Cappelloni I, Di Girolamo M, Maceri F, Vairo G. The influence of implant diameter and length on stress distribution of osseointegrated implants related to crestal bone geometry: a three-dimensional finite element analysis. J Prosthet Dent 2008;100(6):422-431 